\title{
A VLBA 15 GHZ SMALL SEPARATION GRAVITATIONAL LENS SURVEY
}

\author{
A.R. PATNAIK \\ MPIfR, Bonn, Germany \\ M.A. GARRETT AND A. POLATIDIS \\ NRAL, Jodrell Bank, UK \\ AND \\ D. BAGRI \\ NRAO, Socorro, USA
}

\section{Introduction}

We have embarked on a $15 \mathrm{GHz}$ VLBA survey of 1000 flat spectrum sources. We present the results from a 24 hour pilot observing run in which 72 sources were mapped. The primary aims of this project are:

- to search for small separation (1-150 mas) gravitational lens systems

- to identify targets for current $m m$ and anticipated Space VLBI programs

- a morphological classification of compact radio sources at relatively high frequency with sub-mas resolution.

These observations are motivated by the fact that previous radio surveys, using the VLA and MERLIN (e.g. Patnaik et al. 1992), have only been sensitive to image separations $>$ few hundred milliarcseconds (i.e. to lens masses $\left.>10^{9} M_{\odot}\right)$. The aim of this survey is to search for multiply imaged VLBI radio cores with separations $\leq 150$ mas.

\section{Observations and Data Analysis}

In 1995 January 20 we observed 72 sources in a single 24 hour period using 1 thin tape per station (a total of 12 hours of recording/observing time at $64 \mathrm{Mb} / \mathrm{s})$. Each source was observed for four scans of 2 minutes duration (including slew time), spread over $\sim 8 \mathrm{hrs}$ in hour angle, using all $10 \mathrm{VLBA}$ 

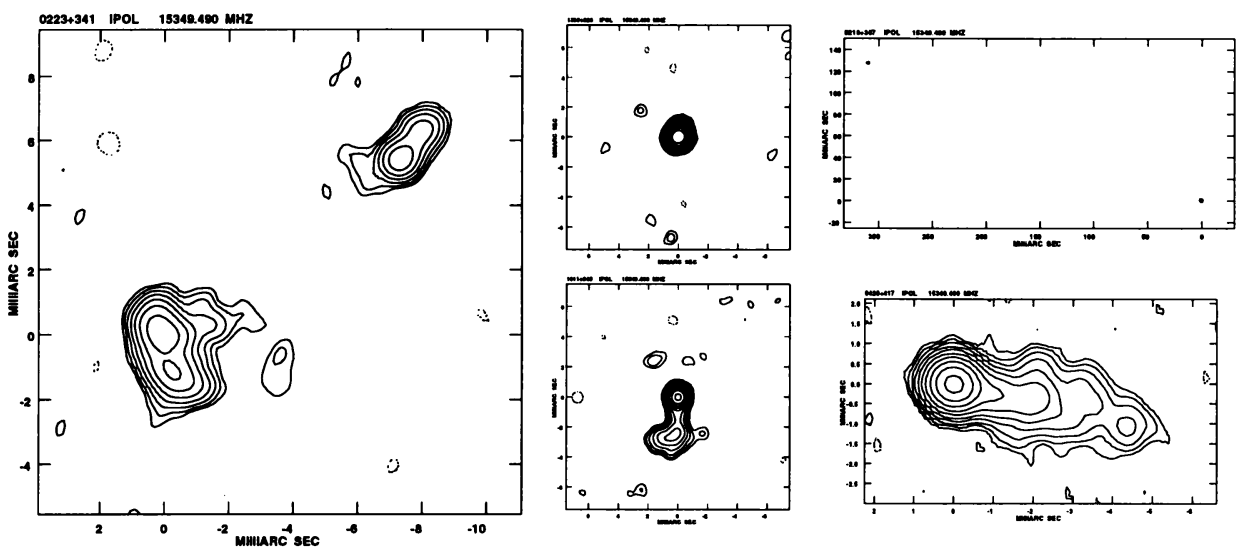

Figure 1. Left: A $15 \mathrm{GHz}$ VLBA map of $0223+341$, contours are spaced by factors of two in brightness, with the lowest at three times the $\mathrm{rms}$ noise level $(0.36 \mathrm{mJy} /$ beam $)$. Right: Examples of (1) one of many excellent Space/mm-VLBI targets, (2) a wide-field map of the known lens system $0218+357,(3,4)$ sources with interesting resolved structure.

telescopes observing at frequency of $15 \mathrm{GHz}$. The data were correlated at the VLBA correlator in Socorro, NM, and were calibrated and mapped using the AIPS package. The map rms noise is typically $\sim 1 \mathrm{mJy} /$ beam.

\section{Results}

Out of 72 sources we observed, 7 are points sources, 24 are point like (i.e. point sources with weak extensions), 24 exhibit typical core-jet structure, 10 are doubles and 4 are triple sources (i.e. 3 separate components, no evidence at the moment if they are one- or two-sided). Three sources were not detected on the intermediate and long baselines. The radio maps and a table of results are available via $f t p: / / j b s s 0 . j b$. man.ac.uk/pub/mikey/survey.ps.

Wide-field maps of all the sources were also made in order to search for additional radio components up to 150 mas from the phase center of the map (see Fig 1).

\section{Best Lens Candidate - 0223+341}

While there are several potential candidates, there is one source which stands out from the rest of the sample $-0223+341$ (see Fig 1). The double structure seen in both the north western and south eastern components is particularly suggestive. The source is identified optically as a "stellar object" but the redshift is unknown. Further observations are planned.

\section{References}

Patnaik A.R., Browne I.W.A., Wilkinson P.N., \& Wrobel J.M., 1992, MNRAS, 254, 655 